\title{
Assistência de enfermagem em núcleo de reabilitação: o papel do enfermeiro
}

\author{
Nursing care in the rehabilitation center: the nurse's role \\ Atención de enfermería en un centro de rehabilitación: el papel de enfermería
}

\author{
Marislei Sanches Panobianco' @ , Isabela de Oliveira Felipel @; Ana Carolina Sipoli Canete' @ ; \\ Larissa Clara Nunes' @ ; Maria Antonieta Spinoso Prado' @
}

'Universidade de São Paulo, Ribeirão Preto, SP, Brasil

\begin{abstract}
RESUMO
Objetivo: reconhecer como mastectomizadas descrevem e compreendem os cuidados de enfermagem que recebem em um núcleo de reabilitação. Método: estudo descritivo, qualitativo; realizado em um núcleo de reabilitação. Participaram 26 mastectomizadas, com 18 anos ou mais, que frequentavam o serviço há um ano, no mínimo, e pelo menos uma vez ao mês. A coleta de dados foi realizada por meio de entrevistas abertas, após aprovação do Comitê de Ética em Pesquisa. Resultados: surgiram quatro categorias: reconhecendo a enfermagem e sua importância no cuidado em saúde de mulheres com câncer de mama; a enfermagem organizando e planejando as atividades assistenciais, sociais e de lazer; a enfermagem liderando o grupo verbal e a enfermagem cuidando e acolhendo. Conclusão: As participantes reconhecem a enfermagem como indispensável na equipe multiprofissional que lhes presta assistência; que são acolhidas e assistidas de forma satisfatória, tanto física como emocionalmente e que isso se deve à organização e gerenciamento de todas as atividades por uma enfermeira.
\end{abstract}

Descritores: Enfermagem em Reabilitação; Neoplasias da Mama; Mastectomia; Papel do Profissional de Enfermagem.

\begin{abstract}
Objective: to recognize how women describe and understand the nursing care they receive in a rehabilitation center following mastectomy. Method: this qualitative, descriptive study was conducted at a rehabilitation center, with 26 patients, aged 18 or over, who had undergone mastectomy and attended the service at least once a month for at least one year. Data were collected by open interviews, after ethics commission approval. Results: four categories emerged: recognizing nursing and its importance in health care for women with breast cancer; nurses organizing and planning care, social and leisure activities; nurses leading the verbal group; and nurses providing care and comfort. Conclusion: The participants recognized that nursing was indispensable to the multi-professional team providing care, that they were welcomed and assisted satisfactorily, both physically and emotionally, and that this was due to a nurse's organizing and managing all activities.
\end{abstract}

Descriptors: Rehabilitation Nursing; Breast Neoplasms; Mastectomy; Nurse's Role.

\section{RESUMEN}

Objetivo: reconocer cómo las mujeres describen y comprenden los cuidados de enfermería que reciben en un centro de rehabilitación tras una mastectomía. Método: estudio cualitativo, descriptivo, realizado en un centro de rehabilitación, con 26 pacientes, de 18 o más años, que se habían sometido a mastectomía y acudieron al servicio al menos una vez al mes durante al menos un año. Los datos se recopilaron mediante entrevistas abiertas, después de la aprobación de la comisión de ética. Resultados: surgieron cuatro categorías: reconocimiento de la enfermería y su importancia en la atención de la salud de las mujeres con cáncer de mama; enfermeras que organizan y planifican actividades asistenciales, sociales y de ocio; enfermeras que lideran el grupo verbal; y enfermeras que brindan atención y comodidad. Conclusión: Los participantes reconocieron que la enfermería era indispensable para el equipo multiprofesional que brindaba los cuidados, que fueron recibidos y atendidos de manera satisfactoria, tanto física como emocionalmente, y que esto se debía a que una enfermera organizaba y gestionaba todas las actividades.

Descriptores: Enfermería en Rehabilitación; Neoplasias de la Mama; Mastectomía; Rol de la Enfermería.

\section{INTRODUÇÃO}

O câncer de mama é o tipo mais comum entre as mulheres, excetuando-se o câncer de pele não melanoma ${ }^{1-3}$. Entre os tratamentos mais utilizados para essa patologia incluem-se a cirurgia, a quimioterapia, a radioterapia e a hormonioterapia. Esses tratamentos podem trazer efeitos deletérios, tanto locais quanto sistêmicos, levando a consequências físicas e emocionais na vida da mulher ${ }^{4,5}$.

Dentre os procedimentos cirúrgicos, a mastectomia consiste na retirada da glândula mamária, podendo causar diversas consequências, entre elas, funcionais, motoras e alteração da autoimagem ${ }^{6,7}$.

Diante de todos os aspectos pós-mastectomia, a mulher necessita de um atendimento envolvendo uma equipe multidisciplinar, a fim de diminuir e tratar possíveis consequências. A atuação do enfermeiro nessa etapa é de extrema importância e envolve alta capacidade para desenvolver atividades educacionais aos pacientes e a seus familiares, assim como para analisar todas as etapas do processo saúde-doença-10. 
Quando nos reportamos ao contexto deste estudo, visa-se um núcleo de reabilitação de mulheres mastectomizadas, que funciona dentro de uma instituição de ensino de enfermagem; criado, coordenado e sob a responsabilidade de enfermeiras.

Assim, o objetivo deste estudo é reconhecer como mastectomizadas descrevem e compreendem os cuidados de enfermagem que recebem em um núcleo de reabilitação.

\section{REVISÃO DE LITERATURA}

Em nosso país, o câncer de mama responde por cerca de $29 \%$ dos casos novos a cada ano, e é também a primeira causa de morte por câncer na população feminina. Para cada ano do triênio 2020-2022 são estimados 66.280 novos casos da doença, mostrando um aumento em relação aos anos anteriores, quando as estimativas foram de 59.700 (2018-2019) ${ }^{1-3}$.

Seus tratamentos dependem do estadiamento do tumor; os mais utilizados são cirurgia, quimioterapia, radioterapia e hormonioterapia, que podem acontecer de forma isolada ou em conjunto e são importantes no controle da doença. No entanto, podem apresentar eventos adversos locais e/ou sistêmicos e podem afetar a vida da mulher ${ }^{4,5}$.

Os procedimentos cirúrgicos utilizados para o tratamento, envolvem a cirurgia conservadora, que consta da extirpação de parte da glândula mamária no quadrante em que o tumor se encontra, e a mastectomia, que consiste da retirada total da glândula mamária, a fim de reduzir as chances de reincidência da doença e melhorar a expectativa de vida ${ }^{11,12}$.

Dessa forma, a mastectomia pode acarretar consequências físicas e emocionais desfavoráveis à vida da mulher; dentre elas, destacam-se as lesões musculares, alterações na sensibilidade, alterações posturais, algias, diminuição ou perda total da amplitude de movimento e da força muscular, perda ou redução da capacidade funcional e linfedema ${ }^{13,14}$.

Nesse sentido, as alterações corporais advindas dos tratamentos podem ser traumáticas para as mulheres, dificultando o manejo da situação vivida; podem levar ainda a um comprometimento emocional, potencializando o momento de fragilidade enfrentado ${ }^{15}$.

Diante disso, a mulher com câncer de mama necessita de reabilitação, ou seja, precisa ser assistida por uma equipe multidisciplinar no intuito de prevenir e/ou detectar, amenizar e tratar essas possíveis complicações.

Quanto à reabilitação, a Organização Mundial de Saúde (OMS) a define como "um conjunto de medidas que assistam pessoas que experimentam ou experimentarão incapacidade para alcançar e manter um ótimo funcionamento em interação com seus ambientes"16.

No que se refere a reabilitar o paciente oncológico, esse processo é considerado fundamental e integrado no tratamento, a fim de reestabelecer os limites impostos pela doença e seu tratamento ${ }^{17}$. Além disso, tal processo integrase ao planejamento terapêutico dos pacientes, mantendo o objetivo da melhora da qualidade de vida e eficácia dos cuidados prestados ${ }^{8,18}$.

Quanto à reabilitação, que deve ser, portanto, multiprofissional e interdisciplinar, tem-se a atuação do enfermeiro como essencial, pois baseia-se em realizar sua avaliação de forma individualizada para se chegar às intervenções de cuidado. Assim, na reabilitação do paciente oncológico, o enfermeiro é um profissional imprescindível, diretamente envolvido em todo o processo ${ }^{8-10}$.

\section{MÉTODO}

Trata-se de um estudo descritivo, com abordagem metodológica qualitativa. Teve como local de pesquisa um núcleo de reabilitação e assistência para mulheres mastectomizadas, vinculado a uma instituição de ensino superior pública do interior do estado de São Paulo.

O serviço de reabilitação tem como objetivo prestar assistência integral à mulher com câncer mamário, e a seus familiares, por meio de uma equipe multidisciplinar; os atendimentos ocorrem três vezes por semana, e já foram atendidas mais de 1.600 mulheres.

A coleta de dados ocorreu entre setembro de 2018 e maio de 2019. Foram critérios de inclusão: mulheres diagnosticadas com câncer de mama, cadastradas neste núcleo de reabilitação e assistência para mulheres mastectomizadas, com idade igual ou superior a 18 anos, no ano da pesquisa, e que frequentavam o serviço no mínimo há um ano e pelo menos uma vez ao mês. Foram critérios de exclusão: mulheres com dificuldades cognitivas para responder à questão da pesquisa. Foram recrutadas 26 mulheres, e todas aceitaram participar.

O convite foi realizado pessoalmente, por uma das pesquisadoras, nos dias e horários de funcionamento do serviço de reabilitação. No momento da coleta de dados, agendada previamente conforme preferência das participantes, foi apresentado às mesmas o projeto de pesquisa, esclarecendo-Ihes os objetivos e a metodologia e, após a assinatura do Termo de Consentimento Livre e Esclarecido (TCLE), foram aplicados um instrumento com perguntas sobre dados pessoais, 
e uma entrevista (de aproximadamente 45 minutos). O critério para término da coleta de dados foi a saturação de dados, ou seja, os dados foram se repetindo e desvendado o fenômeno.

As entrevistas abertas realizadas foram norteadas por perguntas referentes aos cuidados de enfermagem prestados no serviço e sobre a opinião das mulheres em relação a esses cuidados. Novas questões foram acrescentadas com o intuito de esclarecer e fundamentar a experiência. Para caracterização das participantes, foram coletados dados pessoais e clínicos, como: idade; idade no diagnóstico; ocupação; escolaridade; estado civil; religião; tipo de cirurgia; outros tratamentos; data de início no núcleo de reabilitação.

A análise de conteúdo temática foi utilizada para a análise dos dados. A análise de conteúdo se organiza em três etapas: a pré-análise ou organização do material coletado através da homogeneidade, representatividade e importância para responder o problema dessa pesquisa. A exploração do material, que é a codificação e categorização do conteúdo; o tratamento dos resultados obtidos e interpretação, que se refere à interpretação dos dados coletados mediante o uso da inferência ${ }^{19}$

As categorias foram criadas a partir da codificação dos conteúdos das entrevistas, que se mostraram consideráveis para responder o objetivo desse estudo. Os critérios utilizados foram as falas das participantes que se referiam às atividades que eram executadas e de responsabilidade dos profissionais enfermeiros, dentro do núcleo de reabilitação.

Foram elencadas unidades temáticas que se mostraram importantes para identificar como as mulheres que participam de um núcleo de reabilitação descrevem e compreendem os cuidados de enfermagem a elas prestados neste serviço. A seguir, essas unidades foram agrupadas por convergência de significados, classificadas e agregadas em categorias teóricas ou empíricas que comandaram a especificação dos temas ${ }^{20}$.

Após leitura e releitura das entrevistas foram identificadas 15 unidades temáticas que convergiram nas 4 categorias. A primeira categoria, "reconhecendo a enfermagem e sua importância no cuidado em saúde de mulheres com câncer de mama" foi composta por 4 unidades temáticas: reconhecer a enfermagem, trabalho realizado pela enfermagem; recebe cuidados; recebe orientações de cuidados.

A segunda categoria, "a enfermagem organizando e planejando as atividades assistenciais, sociais e de lazer", composta por 3 unidades temáticas: liderança da enfermagem na equipe multidisciplinar; a enfermagem é imprescindível, a importância das atividades sociais e de lazer.

A terceira categoria - "enfermagem liderando o grupo verbal e a enfermagem cuidando e acolhendo", composta das 3 unidades temáticas: a enfermagem comanda o grupo; a enfermagem inspira confiança para o esclarecimento de dúvidas; a enfermagem inspira confiança para a exposição de sentimentos das mulheres.

A última categoria, "a enfermagem cuidando e acolhendo", foi composta de 5 unidades temáticas: sentir-se cuidada, sentir-se acolhida, ser bem tratada, receber carinho e afeição e sentir que os profissionais se preocupam com sua saúde e bem-estar.

Foram respeitados os aspectos éticos contidos na Resolução 466/2012 do Conselho Nacional de Saúde para pesquisas com seres humanos, com autorização do Comitê de Ética em Pesquisa da instituição sob o número de Protocolo CAAE 89824318.0.0000.5393.

\section{RESULTADOS E DISCUSSÃO}

As 26 mulheres entrevistadas para este estudo foram identificadas por M1 (Mulher 1) a M26 (Mulher 26), para preservar suas identidades. Elas tinham idade entre 35 a 78 anos e receberam o diagnóstico de câncer de mama entre 33 e 68 anos. Quanto ao estado civil, 50\% eram casadas, 23\% separadas, 15,4\% viúvas e 11,6\% solteiras. Em relação à ocupação, 50\% das entrevistadas eram do lar. A maioria (77\%) era católica, tinha ensino fundamental incompleto (54\%) e autodeclaravam-se brancas (85\%). Quanto ao tipo de cirurgia, 50\% realizaram mastectomia, 38,4\% quadrantectomia, $3,9 \%$ nodulectomia e $7,7 \%$ quadrantectomia e nodulectomia. No que se refere a outros tipos de tratamento, $77 \%$ das mulheres realizaram radioterapia; 92,3\% fizeram quimioterapia e 61,5\% hormonioterapia, sendo que a maioria realizou os três tratamentos associados.

Analisados os conteúdos das entrevistas, foram identificadas unidades temáticas, as quais foram agregadas em quatro categorias, apresentadas a seguir.

\section{Reconhecendo a enfermagem e sua importância no cuidado em saúde de mulheres com câncer de mama}

Ao se reportarem quanto à forma como entendiam a presença da enfermagem no núcleo de reabilitação, e sobre como reconheciam a enfermagem na equipe de saúde multiprofissional que presta cuidados nesse serviço, as mulheres revelaram que consideram a enfermagem uma peça importante e indispensável nessa equipe: 
É uma equipe, um depende do outro. A enfermagem...como é que eu te falo, a gente acaba tendo dicas das meninas da enfermagem de autoajuda; se não fosse a enfermagem que dá as dicas para a gente, falando o que tem que fazer com o braço, o que pode e o que não pode. (M01)

Olha, eu não sei os nomes muito bem não, mas elas orientam a gente em muita coisa que a gente não conhece, e também as medidas dos braços que são muito importantes para nós, porque conseguimos acompanhar se o braço está inchando ou não. (M07)

Mede nossa pressão, faz a medição do braço, dependendo do grau do linfedema elas passam para as fisioterapeutas que fazem massagem. (M01)

Assim, o que eu vejo é que as meninas que fazem a medição do braço, pesa, vê a pressão, essas meninas eu sei que é da enfermagem. Elas falam para eu não pegar peso, não ficar perto do fogo, de coisa quente, porque pode doer o braço, essas coisas assim, de cuidado com o braço mesmo. (M22)

Dessa forma, elas deixaram claro que, apesar de, algumas vezes não se lembrarem dos nomes do pessoal de enfermagem que as atende no serviço, devido à alta rotatividade de profissionais e alunos na equipe, elas reconhecem que são acolhidas e assistidas de forma a resolverem dúvidas, serem estimuladas ao autocuidado e receberem os cuidados, como por exemplo, aqueles relacionados à prevenção e controle do linfedema, uma complicação séria da cirurgia da mama, e difícil de ser tratada.

Sendo assim, o enfermeiro é um profissional imprescindível na relação entre o paciente oncológico e os outros profissionais da equipe que o atendem ${ }^{8,15}$. Ainda, no decorrer de sua formação e vivência prática, o enfermeiro possui a capacidade de adquirir vasto conhecimento, como também, obter habilidades que lhe conferem a capacidade em lidar com as consequências biopsicossociais que o câncer ocasiona àqueles acometidos, sendo assim, o mesmo é capacitado para realizar atividades para a reabilitação desses pacientes ${ }^{8}$.

Ainda em relação ao cuidado prestado às mulheres, elas citaram outras atividades que reconheciam como sendo de responsabilidade da enfermagem e que eram oferecidas nos dias de atendimento do serviço. Assim, além da realização da perimetria (medida da circunferência dos braços, em vários pontos), destacaram que também recebiam orientações sobre controle de peso, alimentação, automassagem, controle da pressão arterial, glicemia, compondo um cuidado integral à mulher com câncer de mama.

Vale esclarecer que, no serviço de reabilitação, entre as atividades dos atendimentos nos três dias da semana são oferecidos também exercícios físicos direcionados à prevenção e controle das complicações com braço e ombro após a cirurgia e o grupo de discussão (grupo verbal). Além disso, as mulheres podem se beneficiar com atendimento psicológico individual, atividades de terapia ocupacional, atividades educativas, sociais, culturais e de lazer; são realizados encaminhamentos a outros serviços e orientados direitos do paciente com câncer. Mensalmente ocorre uma reunião do Grupo de Família, direcionado aos familiares das mulheres que frequentam o serviço.

\section{A enfermagem organizando e planejando as atividades assistenciais, sociais e de lazer}

As participantes manifestaram-se também em relação a como se organizava o serviço e quem fazia parte dessa organização. Nesse cenário, as mulheres referiram que a organização e gerenciamento do núcleo de reabilitação são realizados por uma enfermeira.

O núcleo presta atividades por meio de uma equipe multiprofissional, mas uma enfermeira contratada para o serviço organiza e gerencia todas as atividades; está presente em todos os dias de atendimento e em todas as atividades internas ou externas ao núcleo, oferecidas para as mulheres. Duas docentes enfermeiras são as responsáveis pelo serviço, sendo que uma delas é coordenadora do núcleo. Os outros profissionais que compõem a equipe são fisioterapeutas, psicólogos, nutricionista e alunos de graduação e pós-graduação.

As participantes percebem que todas as atividades de lazer, palestras, eventos e viagens são planejadas juntamente com a equipe multidisciplinar, porém com a liderança de uma enfermeira.

Elas reconhecem que a enfermagem é imprescindível no serviço e dentro da equipe, quando deixam claro que sem a enfermagem não tem serviço de reabilitação, conforme o último relato a seguir:

É um trabalho em equipe, pois sem a equipe nada funciona. Então a enfermagem participa também da organização dessas atividades. A enfermeira X (enfermeira do serviço) gerencia a organização da atividade, mas tudo é feito a partir da equipe, aí cada um fica responsável por alguma coisa. (M01)

É a X (enfermeira do serviço) que organiza tudo né, ela como enfermeira, então é ela que organiza o serviço pelo meu ponto de vista, corre atrás das coisas, resolve problemas, é tudo ela. (M16)

É a X (enfermeira do serviço) que planeja as atividades, e é feito correto né, porque sempre deu certo né, ela que vai atrás para ver se dá certo, organiza as coisas, é isso. E as meninas também ajudam na organização. (M18)

Ah, eu acho que sem a enfermagem não tem REMA. (M16) 
Diante desses depoimentos, observa-se que uma equipe multidisciplinar tem como objetivo desenvolver um elevado e multifacetado padrão de cuidado, de forma a garantir atenção integral, biopsicossocial e efetiva que exige à reabilitação ${ }^{8,15}$. Além disso, é também papel do enfermeiro, dentro da reabilitação oncológica, voltar-se não só para o cuidado específico com o paciente, mas também com o ensino, coordenação e direcionamento com as próprias pacientes e seus familiares, fazendo com que ele seja o profissional primordial para desempenhar atividades assistenciais irregulares dentro da equipe multidisciplinar ${ }^{8-10}$.

Em continuidade às suas observações sobre os cuidados da equipe multiprofissional do serviço de reabilitação, com ênfase à atuação da enfermagem, as participantes ressaltaram a importância das atividades sociais e de lazer, viagens, almoços, entre outras, de que participam:

No Desfile Rosa, por exemplo, elas (enfermeiras) ajudam a gente a escolher as roupas, leva a gente para almoçar, elas ajudam em tudo, como eu disse é um trabalho em equipe para que o evento aconteça. Elas orientam a gente de como era para fazer, essas coisas. (M01)

Eu acho sim, porque tem muitas mulheres que ficam muito lá para baixo e essas coisas que têm aqui ajuda muito a animar a pessoa, a deixar a pessoa mais animada, mais confiante. Eu acho que participa sim, sempre tem alguém né. (M05)

Eu adoro essas atividades, e é importante, porque levanta o astral da gente, a gente fica mais para cima né, e as meninas da enfermagem ajudam quando tem essas atividades, é um trabalho que eu vejo que é feito em grupo. (M18)

As mulheres aqui entrevistadas mostraram em suas falas, que a enfermagem, especialmente as enfermeiras do serviço de reabilitação em que os dados para esse estudo foram coletados, compreendem a importância do cuidado integral à mulher com câncer de mama, contemplando os aspectos físicos, mas também os psicossociais envolvidos no processo da doença, dos tratamentos e da recuperação.

Autores afirmam que essas atividades fazem grande diferença no tratamento e reabilitação do câncer de mama, principalmente aquelas que trabalham com a autoestima, ressaltando que o tratamento do câncer de mama pode influenciar negativamente na autoimagem e na autoestima da mulher, podendo afetar até mesmo sua sexualidade e solicitar dela uma adaptação frente à situação vivida ${ }^{21,22}$.

\section{A enfermagem liderando o grupo verbal}

Entre as atividades diárias dos atendimentos do serviço de reabilitação, além dos exercícios físicos, acontece o grupo verbal, no qual as participantes discutem questões relacionadas ao enfrentamento do câncer de mama, compartilham suas histórias, dividem suas dúvidas e angústias, mas cabe dizer aqui que elas também aproveitam esse espaço para compartilhar notícias alegres como os bons resultados dos seus exames de acompanhamento, da volta ao trabalho após os tratamentos, entre outros.

E mais uma vez, elas destacam que nesse espaço aparece a enfermeira do serviço na coordenação dos grupos, juntamente com a equipe da psicologia. A enfermeira inspira confiança para o esclarecimento de dúvidas e para a exposição de sentimentos das mulheres que participam desses grupos:

É a X que comanda o grupo, que é enfermeira né. Lá no grupo a gente consegue expressar nossos sentimentos e crescer também né. É a X que gerencia, que orienta e também tem a psicóloga lá, então acho que é um trabalho em conjunto dos dois profissionais. (M02)

Eu consigo sim, as dúvidas são todas respondidas, consigo falar dos meus sentimentos e aquilo que a gente tem mais dúvidas, a X procura profissionais para vim dar palestras sobre aquilo, para orientar a gente. (M04)

Eu gosto do grupo e é a melhor parte, o dia que eu perco o grupo eu fico triste, mas é muito bom o grupo. Ali a gente conversa de tudo, a gente coloca para fora aquilo que está sufocando as vezes, não tem coisa melhor que compartilhar a dor, falar... (M21)

Quem conduz o grupo é a X que é enfermeira né, aí tem as psicólogas também, vejo isso no grupo, que é alguém da enfermagem que comanda. (M22)

Essa relação ímpar entre o enfermeiro e o paciente permite que este profissional seja capaz de ampliar sua assistência, contribuindo no enfrentamento positivo do paciente, como também de seus familiares, em relação à doença e seu processo, além de atuar com práticas e cuidados que reconheçam e respeitem a autonomia, crenças e valores, principalmente, do paciente oncológico ${ }^{8,23}$

Além disso, podemos dizer que outro motivo que evidencia a importância do profissional enfermeiro no processo de reabilitação é sua capacidade de contribuir dentro da equipe multiprofissional, considerando que esse profissional atua em todas as fases do processo de doença e reabilitação, além de auxiliar outras áreas da saúde e na comunidade. Isso resulta em uma construção de conhecimentos, os quais podem ser utilizados no cuidado direto e indireto do paciente, levando assim a uma total reabilitação do indivíduo ${ }^{8,9}$. 


\section{A enfermagem cuidando e acolhendo}

Em continuidade aos depoimentos sobre a equipe de enfermagem no atendimento às participantes deste estudo no núcleo de reabilitação, elas declararam que se sentem cuidadas e acolhidas por esses profissionais. Sentem um cuidado que vai para além da assistência, ou seja, sentem que são bem tratadas e que lhes são oferecidos carinho, afeição e preocupação com seu estado de saúde e seu bem-estar.

Me sinto feliz pelo tratamento, é uma segurança que eu tenho aqui, porque se acontece alguma coisa, elas já me ajudam, me conforta, tiram minhas dúvidas ou procuram alguém que consiga responder. Me sinto muito cuidada. (M01)

Sim, eu me sinto cuidada aqui, no geral eu sou cuidada pelas amigas, pelos profissionais, por todo mundo, aqui tenho amizades sinceras. Eu me sinto cuidada quando eu estou com dor por exemplo, e a $X$ vem e avalia, me fala o que eu devo fazer, então tira aquelas preocupações bestas que a gente coloca na cabeça e outra, eu me sinto cuidada só de vim aqui, sabe, me sinto bem, vindo aqui, é bom demais. (M20)

Sim, eu me sinto cuidada, tudo o que preciso ou tenho dúvida vocês me ajudam, eu sou orientada, me explicam, então eu sou cuidada, e não tem essa de esperar um pouquinho depois eu te atendo, as coisas aqui são na hora que temos dúvida, que você fala. Vocês estão de parabéns (risos). (M26)

Essas falas nos remetem à afirmação de que a enfermagem exerce um papel fundamental nos trabalhos realizados nestes grupos de apoio, pois é papel do enfermeiro ensinar o autocuidado, valorizar o indivíduo, com seus medos e suas dúvidas, visando a promover um crescimento individual a partir da aceitação do indivíduo como ser único e singular, dando-Ihe estímulo e apoio.

$\mathrm{O}$ acolhimento realizado pelo profissional de enfermagem é ressaltado como um fator crucial para a permanência dos pacientes nos grupos de reabilitação ${ }^{15}$, evidenciando a importância do atendimento prestado pelo profissional de enfermagem no processo de reabilitação dos pacientes oncológicos.

\section{Limitações do estudo}

Em relação às limitações deste estudo, houve dificuldade em selecionar as participantes de acordo com os critérios de inclusão e exclusão, visto que algumas delas deixam de frequentar regularmente o núcleo de reabilitação. Outras retornam ao trabalho, mudam de residência para outro município, entre outros.

\section{CONCLUSÃO}

As participantes desse estudo evidenciaram que reconhecem a enfermagem como membro ativo e fundamental na equipe multiprofissional do núcleo de reabilitação, abordando tanto questões físicas como emocionais.

Elas destacaram a presença do enfermeiro no gerenciamento e organização do serviço, além do planejamento de atividades de lazer, sociais e assistenciais. Reconheceram a enfermeira como coordenadora do grupo verbal (juntamente com um profissional de psicologia) e sua importância na participação desse grupo, inspirando confiança para o esclarecimento de suas dúvidas e para a exposição de seus sentimentos. Afirmaram que se sentem cuidadas e acolhidas pelos profissionais de enfermagem.

Assim, um núcleo de reabilitação de mulheres com câncer de mama, que conta com uma equipe multiprofissional, mas que funciona em um espaço dentro de uma escola de enfermagem, foi criado, é coordenado e fica sob a responsabilidade de enfermeiras, é assim reconhecido na voz das mulheres que participam e usufruem do serviço, o que evidencia o sucesso do enfermeiro na gestão de um serviço multiprofissional de saúde. Fica evidente que esse modelo de assistência a mulheres com câncer de mama é cada vez mais necessário e importante, pois poderá lhes proporcionar melhor qualidade de vida, seja qual for a etapa de seu processo de reabilitação.

\section{REFERÊNCIAS}

1. Brasil. Ministério da Saúde. Instituto Nacional de Câncer José Alencar Gomes da Silva. Estimativa 2018 - Incidência de câncer no Brasil [Internet]. Rio de Janeiro, 2017 [cited 2020 Feb 10]. citado em 10 fev 2020https://www.inca.gov.br/imprensa/incaestima-que-havera-cerca-de-600-mil-casos-novos-de-cancer-em-2018.

2. Ministério da Saúde (BR), Instituto Nacional de Câncer José Alencar Gomes da Silva. Estimativa 2020 - Incidência de câncer no Brasil [Internet]. Rio de Janeiro; 2019 [cited 2020 Feb 10]. Available from: https://www.inca.gov.br/publicacoes/livros/estimativa-2020-incidencia-de-cancer-no-brasil.

3. Ministério da Saúde (BR), Instituto Nacional de Câncer José Alencar Gomes da Silva. Estatísticas de câncer [Internet]. Rio de Janeiro; 2020 [cited 2020 Feb 10]. Available from: https://www.inca.gov.br/numeros-de-cancer.

4. Ministério da Saúde (BR), Instituto Nacional de Câncer José Alencar Gomes da Silva. Câncer de mama. [Internet]. Rio de Janeiro; 2020 [cited 2020 May 01]. Available from: https://www.inca.gov.br/tipos-de-cancer/cancer-de-mama. 
5. Lopes WMPS, Figueiredo MLF, Nery IS, Luz MHBA. The knowledge and the use of bra and external prosthesis for mastectomized elderly women. Rev. enferm. UFPE on line. [Internet] 2016 [cited 2020 May 04]; 11(10):4015-20. Available from: https://periodicos.ufpe.br/revistas/revistaenfermagem/article/view/11484.

6. Silva CMC, Vargens OMC. Woman experiencing gynecologic surgery: coping with the changes imposed by surgery. Rev LatinoAm Enfermagem. [Internet] 2016 [cited 2020 Jul 15]; 24:2780. DOI: http://dx.doi.org/10.1590/15188345.1081.2780.

7. Marques LN. Eficácia da estimulação elétrica no tratamento do linfoedema pós mastectomia: uma revisão de literatura [master thesis]. Uberlândia (MG): Faculdade de educação física e fisioterapia, Universidade Federal de Uberlândia; 2019.

8. Teles PA. Atuação do enfermeiro na reabilitação do paciente oncológico: revisão integrativa [master thesis]. Ribeirão Preto (SP): Escola de Enfermagem de Ribeirão Preto, Universidade de São Paulo; 2019.

9. Galdino AR, Pereira LDA, Costa Neto SB, Brandão-Souza C, Amorim MHC. Qualidade de vida de mulheres mastectomizadas matriculadas em um programa de reabilitação. Rev. Pesqui. (Univ. Fed. Estado Rio J., Online). [Internet] 2017 [cited 2020 Feb 09]; 9(2):451-58. Available from: http://www.seer.unirio.br/index.php/cuidadofundamental/article/view/5440.

10. Campos MSV, Rached RDV. O papel da enfermagem na reabilitação física. Int. J. Healthc. Manag. [Internet] 2017 [citado em 14 mai 2020]; 3(1). Available from: https://www.ijhmreview.org/ijhmreview/article/view/116/57.

11. Pereira APVM, Santos GRF, Felipe L, Marianna TF, Luz TFN, Esteves APVS. Mastectomia e mamoplastia na vida das mulheres com câncer de mama. Cadernos de Medicina - Unifeso [Internet] 2019 [cited 2020 May 04]; 2:38-52. Available from: http://www.revista.unifeso.edu.br/index.php/cadernosdemedicinaunifeso/article/view/1294.

12. 12.American Cancer Society. Surgery for breast cancer [Internet]. Atlanta, 2019 [cited 04 may 2020]. Available from: https://www.cancer.org/cancer/breast-cancer/treatment/surgery-for-breast-cancer.html.

13. Rocha JFD, Cruz PKR, Vieira MA, Costa FM, Lima CA. Mastectomia: as cicatrizes na sexualidade feminina. Rev. enferm. UFPE on line. [Internet] 2016 [cited 2020 May 01]; 10(5): 4255-63. Available from: https://periodicos.ufpe.br/revistas/revistaenfermagem/article/view/11171.

14. Silva SH, Koetz LCE, Sehnem E, Grave MTQ. Qualidade de vida pós-mastectomia e sua relação com a força muscular de membros superiors. Fisioter. Pesqui. (Online). [Internet] 2014 [cited 2020 Feb 10]; 21(2):180-85. Available from: http://www.revistas.usp.br/fpusp/article/view/83991.

15. Loyola EAC, Borges ML, Magalhães PAP, Areco FS, Yochimochi LTB, Panobianco MS. Grupo de reabilitação: benefícios e barreiras sob a ótica de mulheres com câncer de mama. Texto \& contexto enferm. [Internet] 2017 [cited 2020 May 01]. 26(1):01-10. Available from: https://www.redalyc.org/articulo.oa?id=71449839003.

16. World Health Organization \& World Bank. World report on disability 2011. World Health Organization [Internet] 2011 [cited 2020 May 01]. Available from: https://apps.who.int/iris/handle/10665/44575.

17. Fretta TDB, Boing L, Bussmann RM. Pain rehabilitation treatment for women with breast cancer. BrJP [Internet] 2019 [cited 2020 May 01]; 2(3):279-83. Available from: https://www.scielo.br/scielo.php?script=sci_arttext\&pid=S259531922019000300279\&lang=pt.

18. Wheelock AE, Bock MA, Martin EL, Hwang J, Ernest M Lou, Rugo HS, et al. SIS.NET: A Randomized Controlled Trial Evaluatinga Web-Based System for Symptom Management AfterTreatment of Breast Cancer. Cancer [Internet] 2015 [cited 2020 May 01]; 121(6):893-9. Available from: https://acsjournals.onlinelibrary.wiley.com/doi/full/10.1002/cncr.29088.

19. Bardin L. Análise de conteúdo. Tradução de Luís Antero Reto e Augusto Pinheiro. Lisboa (PT). 70. ed. Lda; 2011.

20. Minayo MCS. O desafio do conhecimento: pesquisa qualitativa em saúde. 10. Ed. São Paulo: Hucitec, 2007.

21. Gazola C, Bredow D, Pivetta HMF, Braz MM. Percepção de mulheres jovens sobre a sexualidade e a imagem corporal pós mastectomia. Rev. ter. ocup. [Internet] 2017 [cited 2020 May 01]; 28(1):93-9. Available from: http://www.revistas.usp.br/rto/article/view/120708.

22. Paiva ARB, Monteiro CRAV. Qualidade de vida em mulheres mastectomizadas. Revista de investigação biomédica [Internet] 2018 [cited 2020 May 01]; 10(1):30-7. Available from: http://www.ceuma.br/portalderevistas/index.php/RIB/article/view/171.

23. Li J, Huang J, Zhang J, Li Y. A home-based, nurse-led health program for postoperative patients with early-stage cervical cancer: A randomized controlled trial. Eur. J. Oncol. Nurs. [Internet]. 2016 [cited 2020 May 05]; 21:174-80. Available from: https://www.sciencedirect.com/science/article/pii/S1462388915300326?via\%3Dihub. 